\title{
Educación Bilingüe en el Perú: Hitos y Perspectivas
}

\section{Martha Villavicencio Ubillús}

A littie more than a third of Peruvians speak an indigenous languaje. However, the various educational policies have ignored this fact. It was only during the decade of the seventies that some steps were taken toward a bilingual education. In 1987 the General Board of Bilingual Education (DIGEBIL) was created to work towart a new orientation In education, which is bilingual and intercultural, aimed at the peoples who espeak a native language and have a native culture. This model is being put into practice mainly at the primary level.

Un peu plus du tiers de la population péruvienne parle une langue indigène. Cependant, les différentes politiques éducatives ont laissé de côté cette donnée capitale. C' est seulement au cours des années 70 que sont prises certaines mesures visant à une d́ducation bilingue. En 1987 fut crée la Direction Generale d'Education Bilingue (DIGEBIL), qui travaille à une nouvelle orientation de l' éducation destinée aux populations de langue et culture autochtones: une éducation bilingue et interculturelle. De modéle commence a otre mis en oeuvre principalement au niveau primarie. 


\section{Politicas educativas y población indigena*}

\section{en el Perú} tro país.

El multilingüismo y pluriculturalismo constituyen una herencia ancestral en nues-

Según proyeccionés formuladas por la Oficina de-Estadísticas e Informática del Ministerio de Educación en 1989, el Perú tiene 21'255,000 habitantes. En el "Informe de la Polftica Educativa en Marcha. Bases para Sistema Educativo Siglo XXI" se estima que la población total hablante de las 56 lenguas nativas que coexisten en el Perú, suma un promedio de $8^{\prime} 000,000$. De ésta, 214,000 peruanos hablan una lengua amazónica, y por lo tanto más de siete millones y medio hablan una lengua andina (quechua, aymara, hacaru o cauqui).

Sin embargo, pese al número considerable de peruanos que hablan una lengua nativa, en los 167 años de vida republicana, las políticas educativas favorecieron en la práctica la minusvaloración de las lenguas y culturas autóctonas.

En efecto, al establecerse la República, en la segunda década del siglo XIX, se optó por una política asimilacionista. Al respecto, es sumamente elocuente la Constitución de 1933 pues no contiene ninguna referencia a las culturas y lenguas vernáculas, tampoco a la educación para las comunidades rurales ni menos aún a la educación bilingủe intercultural.

Es sólo en el último tercio de este siglo, en el periodo 1972-75 cuando se expiden dispositivos legales que aunque amparados por la Constitución de 1933, fueron dados en el marco de un proceso que pretendió realizar algunos cambios estructurales. Tales dispositivos fueron: la Ley General de Educación y Lineamientos de Política en Educación Bilingüe (1972); el Reglamento de la Educación Básica (1975); el Decreto Ley № 21156 de Oficialización del Quechua (1975); la Resolución Ministerial № 4023 (1975) que aprobó el Alfabeto Básico General del Quechua.

En la Constitución vigente, la posibilidad de una educación bilingüe se vislumbra en el artículo 35 al establecer que "el Estado promueve el estudio y conocimiento de las lenguas aborigenes, Garantiza el derecho de las comunidades quechua, aymara y demás comunidades nativas a recibir educación primaria también en su propio idioma o lengua".

\footnotetext{
- El término "indígena" designa a los descendientes de los pobladores originarios de América, es decir aquellos que estaban establecidos antes de la llegada de los espafoles, que mantienen su lengua y cultura.
} 
Los artículos 2, 34, 83 y 161 de la Constitución actual dejan abierta la posibilidad al Estado o más bien la obligatoriedad de implementar una educación bilingüe, así como de realizar acciones destinadas a la conservación y desarrollo de las leguas y culturas vernáculas.

En los artículos 3, 4 y 5 de la Ley General de Educación № 23384 (1982) se hace referencia a la atención a los sectores marginados, a la revaloración de las culturas vernáculas y a la identidad nacional. En el artículo $4^{\natural}$, en el capítulo referente a la Educación Primaria, se precisa: "En las comunidades cuya lengua no es el castellano se inicia esta educación en la lengua autóctona con tendencia a la castellanización progresiva a fin de consolidar en el educando sus características socioculturales con las que son propias de la sociedad moderna". Así mismo, en el artículo 45 del capítulo relacionado con la al fabetización, se dice: "La alfabetización se cumplirá en forma selectiva y progresiva, y es impartida preferentemente, en la lengua materna en las comunidades de lengua vernácula, integrada en un proceso de castellanización".

Los esfuerzos traducidos en programas educativos para "castellanizar" en poblaciones vernáculo-hablantes, en el Perú y en otros países de Latinoamérica, no han dado el resultado esperado.

La educación para castellanizar no ha contribuido a solucionar la aguda problemática socio-económica y bajo nivel educativo de las poblaciones de culturas autóctonas.

La educación en las poblaciones indigenas ha venido propiciando un bilingüismo sustractivo ya que el aprendizaje del castellano ha conllevado ya sea a la pérdida progresiva de la lengua materna o a un menor uso de ésta, relegándola a funciones menos importantes. El currículo centrado en el profesor mas no en el alumno, no ha considerado sus intereses ni las necesidades de su contexto social, lingüistico y cultural.

La educación ha contribuido así a la alienación de la población vernáculo-hablante, desarraigándola de su espacio y bloqueando el desarrollo de su capacidad creativa, entre otras causas, debido a la falta de importancia explícita de su lengua y cultura en el currículo oficial.

Las poblaciones vernáculo-hablantes tienen estructuras políticas y socioeconómicas autóctonas, que es necesario comprender, valorar y difundir. Estas poblaciones además de distinguirse por su lengua se diferencian entre otros rasgos por su organización sociocultural, valores, creencias, conocimientos, que constituyen patrimonio del pafs y que tradicionalmente no han sido tomados en cuenta, excepto en proyectos educativos experimentales sobre todo a partir de la década del 70 .

El déficit educativo en estas poblaciones de lengua y cultura autóctonas es alarmante. El porcentaje de analfabetos excede al promedio del país; es allí donde se dan las más altas tasas de bajo rendimiento escolar, repetición y deserción. Y en el caso de aquellos que han concluido todas las fases de escolarización, generalmente viven una crisis de identidad a causa de la educación que recibieron. 


\section{La educación billiagüe intercultural en la política educativa de 1987 - 1989}

El Ministerio de Educación ha priorizado la atención educativa de la población indígena creando la Dirección General de Educación Bilingủe en diciembre de 1987 y también ha formulado lineamientos de política que orientan una nueva educación para la población de lengua y cultura autóctonas; nueva educación que se caracteriza por ser bilingüe e intercultural.

La nueva Política Nacional de Educación surge del análisis de la realidad del Perú contemporáneo y del imperativo de superar esa realidad.

La nueva Política Educativa precisa que el fin fundamental de la Educación es la formación de la conciencia histórica nacional. La conciencia histórica se conceptúa como la capacidad de las personas para reconocerse a sí mismas como partes integrantes de su comunidad e identificarse con ella.

De ese fin fundamental se derivan tres fines especificos:

a) Educar para la democracia

b) Educar para el desarrollo, y

c) Educar para la cultura

En lo que específicamente se refiere a las poblaciones de lengua y cultura autóctonas:

- Educar para la democracia, garantiza superar la incomunicación posibilitando una educación bilingüe para la población vernáculo-hablante, que rescate, aproveche y revalore las lenguas nativas. Asimismo asegura la capacidad de entender la naturaleza de los problemas políticos orientándose en el tiempo y en el espacio, y conociendo el origen de sus problemas y proyecciones futuras.

- Educar para el desarrollo, contribuye al cambio de la estructura social.

- Una educación para la cultura, respeta las singularidades de los pueblos para armonizarlos en una cultura nacional.

En este sentido, entre los principios de la Educación Peruana de la actual Política Educátiva se incluye "el reconocimiento de la pluralidad cultural y el multilingüismo del Perú y el respeto de las identidades de las comunidades campesinas y nativas en el proceso de integración nacional". 
Asimismo uno de los Objetivos de la Educación Peruana es:

"La valoración, uso y difusión de las lenguas vernáculas y el empleo del castellano como lengua común".

Es en el contexto de esta Política Educativa que se define la educación bilingüe 'Intercultural y se formulan los lineamientos de política respectivos.

La Educación Bilingüe Intercultural se concibe como aquella que, además de formar sujetos bilingües con óptima competencia comunicativa en su lengua materna y en castellano, posibilita la identificación con su cultura de origen y el conocimiento de otras culturas minoritarias y mayoritarias. $Y$ en base a una actitud de respeto y reflexión, permite al educando discernir, decidir y actuar respecto a los elementos de otras culturas que podrían constituir un valioso aporte para el mejoramiento cualitativo de su nivel de vida y de su comunidad, enriqueciendo así su propia cultura. Con esto se aspira a equiparar las posibilidades, a entender el orgullo por la cultura propia y comentarlo, y a posibilitar que el educando se oriente entre las otras culturas con seguridad y confianza en sí mismo.

La educación bilingüe intercultural tiene como objetivos generales:

Coadyuvar al logro de una identidad nacional caracterizada por la conciencia de un país unido en la diversidad.

Contribuir al rescate, revaloración y desarrollo de las lenguas y culturas autóctonas, evitando su deterioro y desaparición.

Formar sujetos bilingües con una óptima competencia comunicativa en su lengua materna y en castellano como segunda lengua.

Esto implica el manejo oral y escrito de habilidades receptivas y productivas, en las dos lenguas.

Posibilitar la comunicación y entendimiento entre todos los peruanos a través del castellano como lengua común, y la comprensión de los códigos culturales diferentes a los de la propia cultura.

Desarrollar en el poblador de habla vernácula su sentimiento de autoestima que posibilite su seguridad en sí mismo para contribuir en la construcción de una sociedad libre, justa y solidaria.

Contribuir en la institución de la paz positiva en la sociedad peruana.

La Educación Bilingüe Intercultural constituye una modalidad que se da a través de los diferentes niveles del sistema educativo peruano. 


\section{El reto de la implementación de la educación bilingüe intercultural*}

Traducir en la praxis educativa la concepción de EI3I para el logro de los objetivos formulados, en los 56 grupos hablantes de lenguas indigenas, es evidente que constituye un gran reto para el Estado Peruano.

El abordaje de la implementación de la Educación Bilingüe Intercultural ha supuesto el planteamiento de una estrategia de aplicación gradual, a ejecutar en el marco del sistema educativo formal, en base a la articulación de los programas y proyectos de educación bilingüe que han venido ejecutándose aisladamente, sobre todo a partir de la década del 70, tanto en el área andina como en la Amazonía.

La estrategia diseñada por la Dirección General de Educación Bilingüe (DIGEBIL), implica la aplicación progresiva de la modalidad de Educación Bilingüe Intercultural (EBI) en los diferentes niveles del sistema educativo: Educación Inicial, Educación Primaria, Educación Secundaria y Educación Superior. Tal estrategia tiene fundamentalmente tres etapas:

a) Experimentación de la Educación Bilingüe en centros educativos seleccionados.

b) Expansión de la Educación Bilingüe en centros educativos seleccionados y

c) Generalización en todos los centros educativos que requieren de Educación Bilingüe Intercultural.

Considerando que el mayor avance en la sistematización de metodologías y técnicas para la EBI se tiene en el nivel de Educación Primaria Bilingủe, en 1989, en algunos departamentos con población vernáculo-hablante, se ha iniciado ya la etapa de expansión en escuelas de Educación Primaria Bilingüe; mientras que en los otros niveles -Educación Inicial, Educación Secundaria y Educación Superior - aún se está en la etapa de experimentación.

A 1989, se imparte Educación Bilingüe en 28 lenguas autóctonas, a través de 2,375 centros educativos, como se indica en el cuadro siguiente:

- Educación Bilingie Intercultural = EBI. 


\begin{tabular}{|c|c|c|c|c|c|c|}
\hline \multirow[t]{3}{*}{ AREA } & \multirow{3}{*}{$\begin{array}{l}\text { ND LENGUAS } \\
\text { AUTOCTONAS }\end{array}$} & \multicolumn{5}{|c|}{ N'DE CENTROS EDUCATIVOS / PROGRAMAS EN: } \\
\hline & & \multicolumn{2}{|r|}{ E.I. } & E.P. & E SEC. & E. SUP \\
\hline & & CEI & PRONOEI & CEP & CES & $\mathrm{CFM}$ \\
\hline ANDINA & 2 & 02 & $\rightarrow$ & 1652 & 02 & 2 \\
\hline $\begin{array}{l}\text { AM A ZO- } \\
\text { NICA }\end{array}$ & 26 & 36 & 79 & 583 & 17 & 2 \\
\hline TOTAL & 28 & 38 & 79 & 2235 & 19 & 4 \\
\hline
\end{tabular}

\section{Educación Primaria Billingủe}

De 1988 a 1989, la atención en Educación Primaria Bilingüe se ha incrementado en un $50 \%$ a nivel del número de escuelas en que se aplica la modalidad educativa, pasando de una matrícula aproximada de 26,820 a 64,000 alumnos. Esto significa que sólo se ha brindado EBI al $8.4 \%$ del total estimado de educandos de primaria que la requieren.

El mapa siguiente ilustra la distribución de las escuelas en que se aplica la Educación Primaria Bilingùe, a nivel nacional, en el marco de las fases de experimentación y expaņsión.

La aplicación de la EBI en cualquiera de las fases de la estrategia referida, requiere de la capacitación sistemática de los docentes en servicio, en "Fundamentos y Tecnología para la Educación Bilingüe Intercultural". Asimismo, es necesario la disponibilidad de material educativo adecuado, tanto en Lenguaje en Lengua Materna (L1), como en castellano como Segunda Lengua (L2), que sirva de apoyo al proceso de enseñanza-aprendizaje.

En este sentido, se ha realizado cursos de Capacitación Docente y también, en el transcurso de 1989, se han impreso 21,400 ejemplares entre libros, láminas, cuadernos y guías para Educación Primaria Bilingüe. Entre este material educativo se incluye el Módulo de Primer Grado EBI para el Trapecio Andino: Libro "KUSI" y cuademo "KUSI" para el aprendizaje de la lecto-escritura en Quechua; libro "SAPITO" para Castellano como Segunda Lengua; y cuaderno de Matemática "YUPASUN". Este módulo ha sido adecuado tomando como base los libros del mismo nombre que se validaron en el Programa de Educación Bilingüe de Puno en el período 1980-1985. En 1986-88 se aplicaron por primera vez experimentalmente en centros educativos del Trapecio Andino, en el marco del Proyecto Perú BIRF II a cargo del INIDE; y luego de un reajuste, se aplican otra vez en 1989, a nivel experiemental, en 35 escuelas de los departamentos de Cusco, Arequipa, Moquegua, Ayacucho y Huancavelica; con la perspectiva de disponer de un módulo validado aplicable para el Primer Grado de Educación Primaria Bilingủe Intercultural en el Sur Andino. 


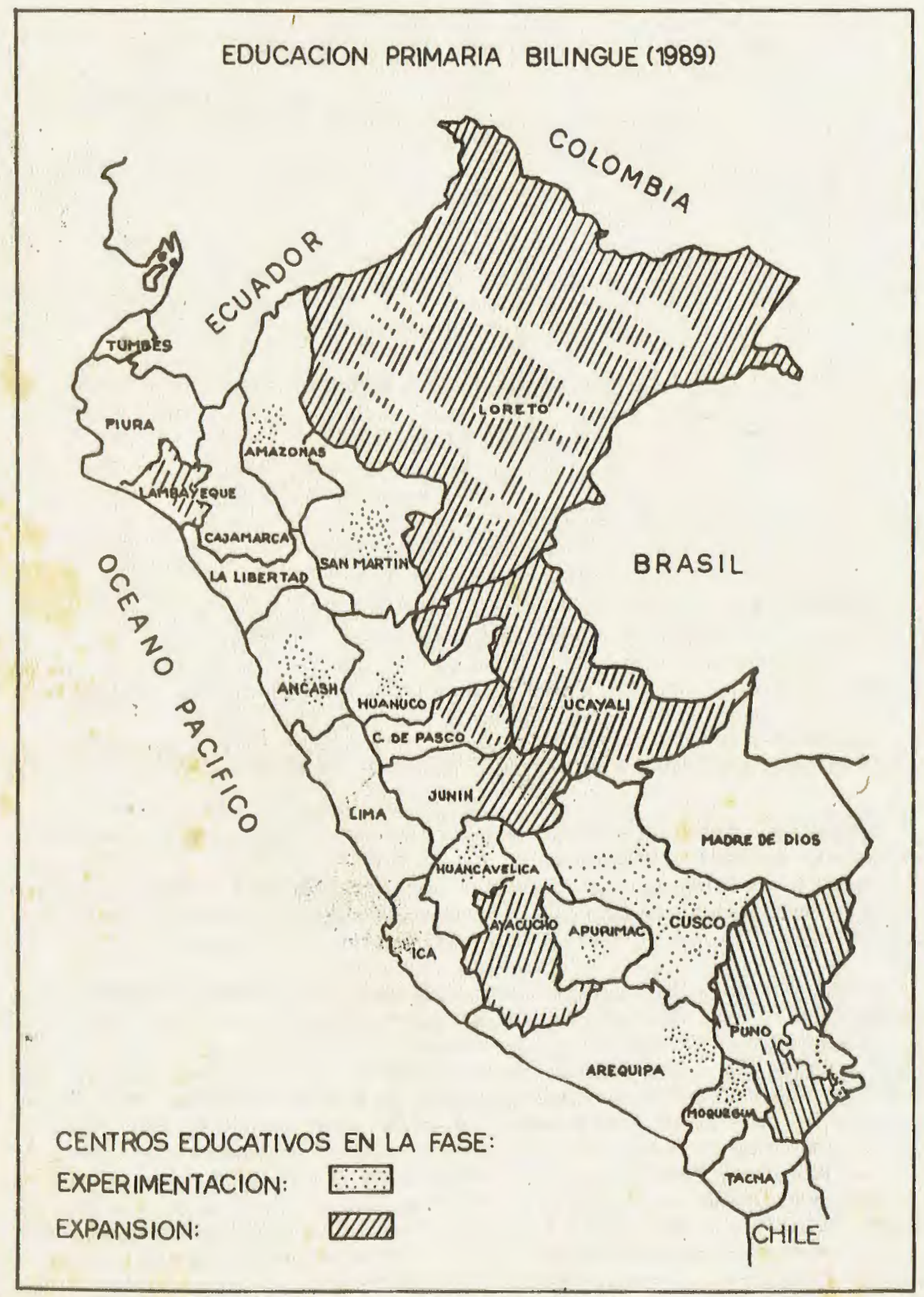

Fuente: Dirección General de Educación Bilinguje 
En 1989 también se ha producido e impreso el Programa Curricular pará Primer Grado de Educación Primaria Bilingüe. Los programas curriculares para todos los grados de Educación Primaria Bilingüe también ya han sido elaborados con participación de docentes vernáculo-hablantes y especialistas y se encuentra en proceso de revisión, para su impresión y aplicación experimental a partir de 1990. También se ha preparado la Guía para la Evaluación del Educando de Educación primaria Bilingüe, la Libreta del Educando y el Registro de Evaluación del Educando de Educación Primaria Bilingüe de Menores.

En los programas curriculares de Educación Primaria Bilingüe Intercultural se incluyen dos líneas de Acción Educativa de Lenguaje: Lenguaje en Lengua Materna y Castellano como Segunda Lengua: la Lengua Materna (L1) se usará y desarrullará en forma dosificada en todos los grados de la escolaridad, en el desarrollo de las diferentes líneas de acción educativa. Castellano como Segunda Lengua (L2) se enseñará con la metodología adecuada a las condiciones particulares que presenta el castellano en relación a la lengua materna de los educandos vernáculo-hablantes; y también se usará en forma gradual y dosificada en cl desarrollo curricular de las otras líneas de acción educativa.

La programación curricular para cada grado de Educación Primaria Bilingüe se efectuará en tres niveles: Nacional, Regional y Microrregional.

\section{Educación Superior}

Después del nivel de Educación Primaria, es en Educación Superior en que a partir de 1985 se vienen logrando algunos avances en lo que se relaciona con Educación Bilingüe.

En efecto, hay dos Institutos Superiores Pedagógicos:

El ISP de Puno en el Sur Andino, y el ISP Bilingüe de Yarinacocha en la Amazonía, que forman profesores y profesionalizan docentes de Educación Primaria Bilinguie desde 1985.

Asimismo, en el compromiso para la búsqueda de propuestas alternativas que respodan a la urgencia del mejoramiento cualitativo de la educación de la población hablante de lenguas autóctonas, particularmente en las zonas rurales, se suman:

- El Instituto Superior Pedagógico de Iquitos que desde 1988 ha iniciado la ejecución experimental del "Programa de Formación de Maestros Bilingües de la Amazonía Peruana", en base a un Convenio suscrito por el Ministerio de Educacion, la AIDESEP (Asociación Interétnica de Desarrollo de la Selva Peruana), la CORDELOR y el Centro de Cooperación Internacional TERRA NUOVA; y

- El Instituto Superior Tecnológico Pedagógico de Urubamba, que desde 1989 desarrolla un Programa Experimental de Profesionalización de docentes para las Areas Rurales Andinas, en el cual se incluye los componentes de educación Bilingüe e interculturalidad. 
En la línea de formación de recursos humanos de alto nivel, se inscribe la Escuela de Post-Grado en Lingüística Andina y Educación de la Universidad del Altiplano de Puno en la que durante 1986-1989 se han desarrollado dos programas, uno de Segunda Especializa* ción y otro de Maestría.

\section{Perspectivas}

A fin de apoyar el desarrollo de la Educación Bilingüe Intercultural en el país y por ende el mejoramiento de la calidad educativa del servicio que se brinda a la población vernáculo hablante, hay dos tareas prioritarias a realizar:

a) La profundización y sistematización del conocimiento de las culturas de cada grupo etno-lingüístico y

b) La planificación del lenguaje en las lenguas indígenas, que implica decisiones no sólo sobre sus alfabetos y ortografía, sino también sobre normalización y estandarización, específicamente del lenguaje en el campo técnico-pedagógico.

Estas dos bases son fundamentales, pues de ellas se nutre la operativización de la Educación Bilingủe Intercultural para plasmarse en la realidad de acuerdo a su concepción.

El desarrollo de la Educación Bilingủe Intercultural ha de darse no sólo a través de los diferentes niveles del sistema educativo formal sino que también ha de implementarse a nivel no formal, a fin de contribuir al logro de sus objetivos.

Asimismo, la educación en general deberá incluir sistemáticamente actividades cuyo propósito sea que los peruanos adopten una actitud positiva hacia el plurilingüismo y multiculturalismo que caracterizan a nuestro pais ya que estos constituyen una riqueza. Un estado multilingủe dispone de tantas vías alternativas para el desarrollo cuantas lenguas o culturas tiene en su interior.

Finalmente, es necesario considerar que la mayor o menor celeridad en el desarrollo de la Educación Bilingüe Intercultural en el futuro, estará en función de los recursos financieros que se asigne para su implementación, que a su vez dependerá de la voluntad política de los futuros gobiernos. 